\title{
Toracotomía posterior: doble abordaje vertebral torácico con incisión única
}

\author{
Posterior thoracotomy: a two-step spinal thoracic approach \\ Toracotomia posterior: dupla abordagem vertebral torácica com \\ incisão única
}

\author{
Mariano Augusto Noel' \\ Romina Maria Rosa Corrado' \\ Carlos Alberto Tello' \\ Eduardo Galaretto' \\ Alejandra Francheri Wilson' \\ Ernesto Bersusky'
}

\section{RESUMEN}

Introducción: durante los últimos diez años, hemos utilizado la toracotomía posterior para abordar la porción superior del tórax en procedimientos combinados anteriores $\mathrm{y}$ posteriores. Actualmente hemos extendido esta indicación a toda la columna torácica en remplazo de la toracotomía convencional y toracoscopía. Objetivo: evaluar el rango de posibilidades y complicaciones asociadas con este nuevo abordaje, que permite combinar la cirugía vertebral torácica anterior y posterior con la misma incisión cutánea posterior. Métodos: fueron evaluados, retrospectivamente, 35 pacientes operados entre los años del 2003 y 2007. En todos se realizó doble abordaje, combinando una toracotomía posterior y abordaje posterior, mediante una sola incisión medial, para diferentes objetivos: descompresión medular, artrodesis, osteotomías o vertebrectomías. Se evaluaron los valores angulares, las etiologías, la edad, los niveles vertebrales, la cantidad de toracotomías y las complicaciones. Resultados: hubo un promedio de edad de 14,1

\section{RESUMO}

Introdução: nos últimos dez anos, foi utilizada a toracotomia posterior para a abordagem da porção superior do tórax em procedimentos combinados anteriores e posteriores. Atualmente, esta indicação tem sido estendida por toda a coluna torácica ao invés da toracotomia convencional e da toracoscopia. Objetivo: avaliar as possibilidades e complicações associadas a esta nova abordagem, que permite combinar cirurgias em vértebras torácicas anterior e posterior com a mesma incisão cutânea posterior. Métodos: foram avaliados, retrospectivamente, 35 pacientes operados entre 2003 e 2007. Em todos eles, realizou-se dupla abordagem combinando uma toracotomia posterior e abordagem posterior, feita só uma ferida cirúrgica, para diferentes objetivos: descompressão medular, artrodese, osteotomias, ou vertebrectomias. Foram avaliadas: as magnitudes dos ângulos, a etiologia, a idade, os níveis vertebrais, a quantidade de toracotomias e as complicações. Resultados: média de idade de 14,1 anos (1-65), 10 cifoses com média

\section{ABSTRACT}

Introduction: over the last ten years, for patients who needed a combined anterior-posterior approach, an alternative thoracotomy has been used by posterior approach using in the second step the same posterior mid-line skin incision as was used in the first step. Objective: to assess the range of possibilities and complications associated with this new approach, which allows to mix a two-step surgery through a single posterior skin incision. Methods: thirty-five patients operated between 2003 and 2007 were evaluated. All patients underwent a twostep approach through a single posterior mid-line skin incision for spinal cord decompression, discectomy, arthrodesis, osteotomy, or vertebrectomy. The angular magnitudes, etiology, age, vertebral levels, number of thoracotomy, and complications were evaluated. Results: mean age 14.1 years (165 years old), ten kyphosis, and 24 kyphoscoliosis. Mean scoliosis was $80.5^{\circ}\left(60-105^{\circ}\right)$, mean kyphosis was $96.8^{\circ}\left(76^{\circ}-131^{\circ}\right)$. Etiology: genetic

Trabajo realizado en el Hospital Nacional de Pediatria Prof. Dr. Juan P. Garrahan - Buenos Aires, Argentina.

1Hospital Nacional de Pediatria Prof. Dr. Profesor J. P. Garrahan - Buenos Aires, Argentina. 
años (1-65), diez cifosis, valor promedio 96,8 (76-131); 24 escoliosis valor promedio 80 (60-105). Etiología: síndromes genéticos, 11; escoliosis idiopática, 6; neurológicas, 5; congénitas, 4; tumores, 4; fracturas, 2; hernia discal, 1; infección, 1. Toracotomía de 1 nivel 30 doble 5 . Nivel superior T3 e inferior T10. Complicaciones: 1 hemotórax y dos infecciones de herida. Conclusión: esta vía permite acceder a todo nivel torácico en procedimientos combinados mediante una sola incisión cutánea. de 96,8 (76-131); 24 escolioses com média de $80^{\circ}$ (60-105). Etiologia: síndromes genéticos, 11; escolioses idiopática, 6; neurológicas, 5; congênitas, 4; tumores, 4; fraturas, 2; hérnia de disco, 1; infecção, 1; toracotomia de 1; nível 30, duplo 5; nível superior t3 e inferior t10. As complicações observadas foram um paciente com hemotórax e dois com infecções por causa da ferida. Conclusão: esta via permite o acesso a todo o nível torácico em procedimentos combinados mediante só uma ferida cirúrgica.

\author{
DESCRITORES: Toracotomia/ \\ métodos; Toracotomia/ \\ complicações; Procedimentos \\ cirúrgicos operatórios/ \\ métodos
}

syndromes, 11; idiopathic scoliosis, 6; neurological, 5; congenital, 4; fractures, 2; disc herniation, 1; tumors, 4; infection, 1. Thoracotomy was single in 30 and double in 5, and highest at level T3 and lowest T10. The complications that occurred was one pleural hemorrhage and two infections of the posterior surgical wound (8.6\%). Conclusion: this approach allows to accede at all the levels of thoracic previous in procedures combined through a single posterior skin incision.

\section{INTRODUCCIÓN}

Desde principios del siglo 1960, Hogdson and Stock $^{1}$ dan popularidad al abordaje anterior de columna, ampliándose rápidamente la frecuencia de sus indicaciones y objetivos. Posteriormente, se fueron desarrollando nuevas alternativas técnicas de abordaje $\mathrm{e}^{2-7}$.

Los objetivos de la vía anterior para columna torácica son en general: resección discal, descompresión medular, osteotomías, resecciones vertebrales, drenajes, tomas biopsias, estabilizaciones instrumentadas, aporte de injertos y correcciones angulares, aplicadas a distintas variantes de patologías infecciosas, degenerativas tumorales o traumáticas.

El abordaje vertebral anterior torácico está frecuentemente asociado a un procedimiento vertebral posterior complementario o principal, secuencial o simultáneo e implica casi siempre dos abordajes mayores y dos o más incisiones cutáneas.

La necesidad de movilizar la escápula en abordajes torácicos altos y seccionar la musculatura peri-escapular con la consiguiente morbilidad, motivó nuestra búsqueda y diseño de un abordaje alternativo eficiente y con poca agresión, al que denominamos toracotomía posterior. Luego de su aplicación inicial acotada, se comenzó a aplicarlo a todo nivel, edad y condición en columna torácica anterior en reemplazo de la toracotomía, costotransversectomia y toracoscopia videoasistida.

\section{MÉTODOS}

Para esta evaluación, fueron seleccionados, retrospectivamente, 35 pacientes operados por nuestro equipo entre los años 2003 y 2007 que hubiesen sido sometidos a un doble abordaje de columna torácica utilizando la toracotomía posterior como acceso a la columna anterior, y la misma incisión cutánea para el acceso al raquis posterior, admitiendo en la muestra todos los diagnósticos, objetivos del procedimiento y edades. En todos ellos se evaluó la edad, el diagnóstico etiológico, el tipo y magnitud de la deformidad previa (cifosis o escoliosis), extensión superior e inferior del procedimiento anterior, cantidad de toracotomías y complicaciones postoperatorias vinculadas al abordaje (ambos) durante el periodo postoperatorio de la internación y como mínimo seis meses posteriores.

La técnica de la toracotomía posterior es la siguiente:

- Posición en decúbito lateral estricto (como para toracotomía convencional);

- Campos dejando expuesto hemitórax hasta hombro superior y excediendo la línea media posterior;

- Incisión en línea media posterior al menos entre T3 a T10 hasta apófisis espinosas (Figura 1);

- Desinsersión medial de trapecio y romboides entre C5 y T12;

- Movilización lateral de flap cutáneo, músculos y escápula (Figuras 2 y 3);

- Exposición del plano costal posterior y elección de costilla o espacio intercostal de acceso (Figura 4);

- Remoción de 7 a $10 \mathrm{~cm}$ de costilla, incluyendo porción costovertebral respetando la musculatura paravertebral y transversa (Figura 5);

- Colocación de separador costal con exposición del área y realización del objetivo específico (Figura 6);

- Cierre con drenaje bajo agua. Se aproximan las costillas con puntos fuertes peri o transcostales mediante simple ajuste manual, no suele ser necesario el aproximador costal si se reseco el fragmento costal incluyendo todo su extremo posterior permitiendo su ajuste manual sin asistencia.

- Reinserción medial de trapecio y romboides en línea media; 


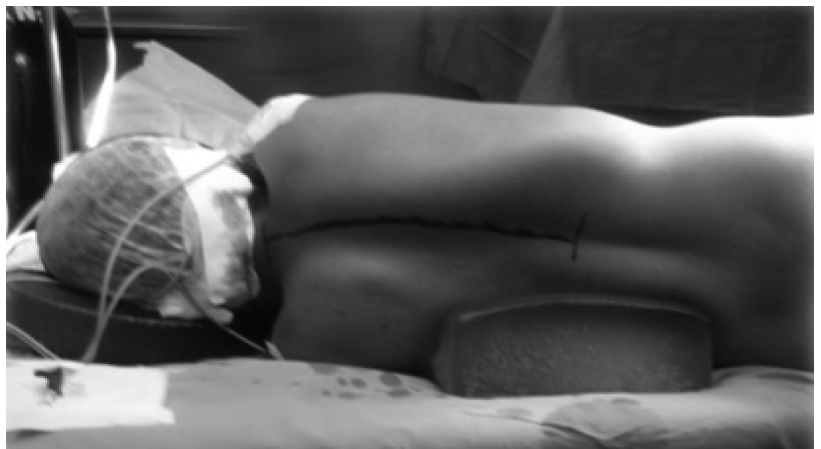

Figura 7

Posición: decúbito lateral estricto (como para toracotomía convencional). Incisión en línea media posterior al menos entre T3 a T10 hasta apófisis espinosas.

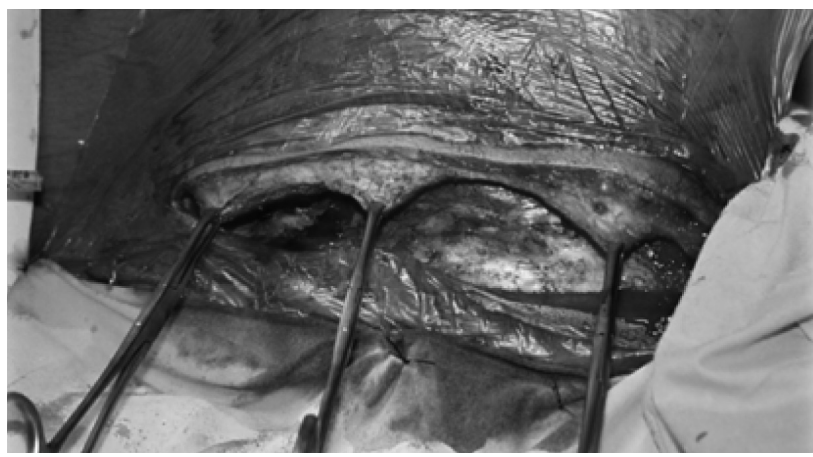

Figura 2

Desinserción medial de Trapecio C5 - T12.

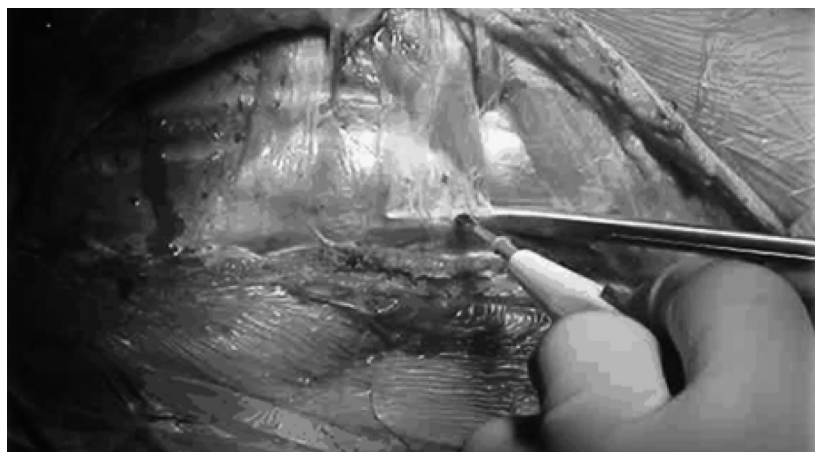

Figura 3

Desinserción medial del romboide de C5 - T12.

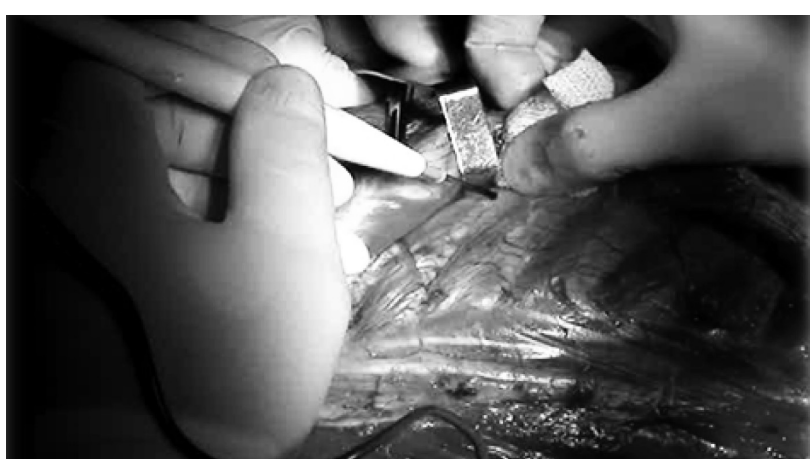

Figura 4

Exposición de plano costal posterior y elección de costilla o espacio intercostal de acceso.

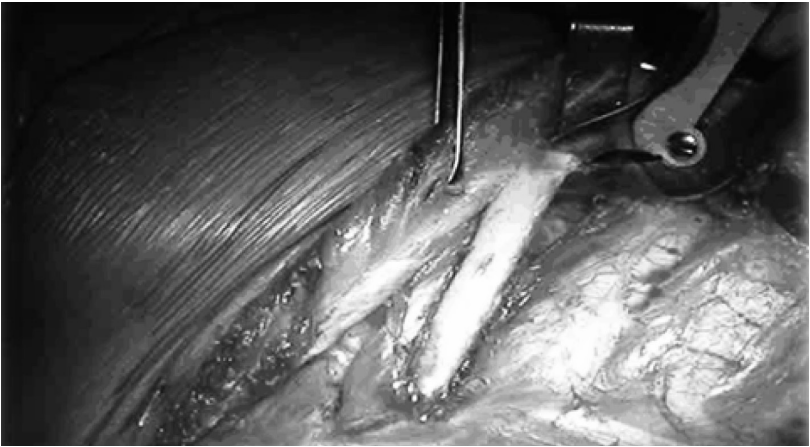

Figura 5

Remoción de 7 a $10 \mathrm{~cm}$ de costilla incluyendo porción costovertebral respetando musculatura para vertebral y transversa.
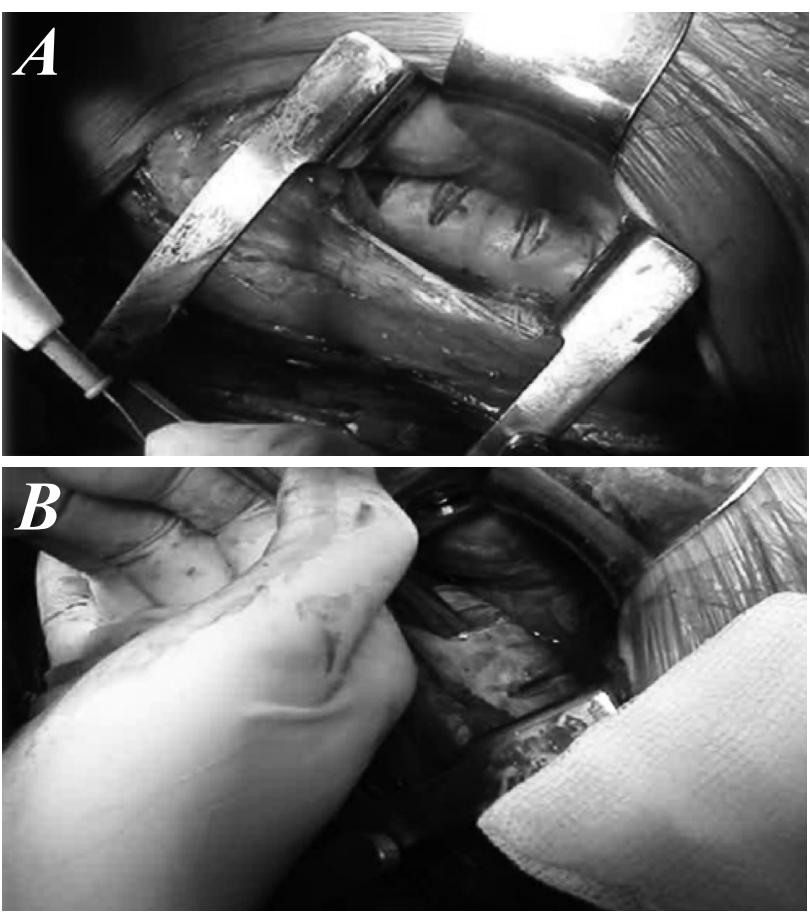

Figura 6

A Colocación de separador costal exposición del área y realización del objetivo especifico.

- Cuando el procedimiento es complementario al posterior (como en todos estos casos), se realizó un cierre provisorio de la herida, lo cubrimos con un campo adhesivo estéril y recolocamos al paciente en decúbito prono como para acceder al tiempo posterior con confort, realizando la reinserción final del trapecio y romboides al final de ambos los procedimientos (Figuras 7 y 8 ).

\section{RESULTADOS}

La edad de los pacientes osciló entre 1 a 65 años con un promedio de 14,1 años; la escoliosis como deformidad principal estuvo presente en 24 casos con valores angulares de entre 60 y 105 grados con promedio de 80 grados; la cifosis como deformidad principal se presentó en diez casos con valores angulares entre 76 y 131 grados y promedio 96,8. La etiología 


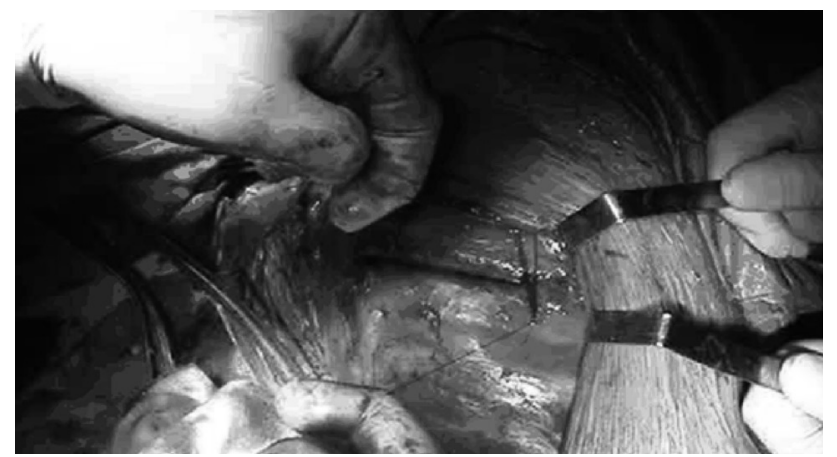

Figura 7

En general el aproximador costal no es necesario cuando la cabeza costal es resecada.

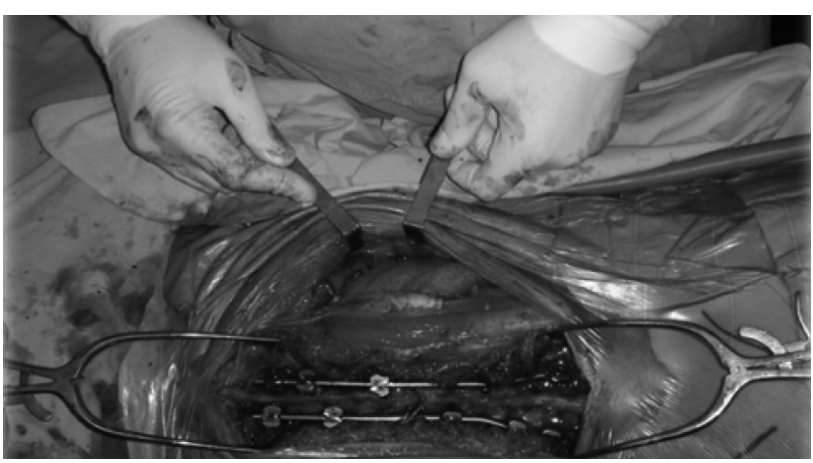

Figura 8

Fin de ambos procedimientos.

fue genética en 11 casos, escoliosis idiopática en 6 casos, neurológica en 5 casos, congénita en 4 casos, tumores en 4 casos, fracturas en 2 casos, hernia discal dorsal en 1 caso e infección en 1 caso (Gráfico 1). La toracotomía fue única en 30 casos y doble en 5 casos. Las complicaciones asociadas a la vía fueron tres $(8,5 \%)$ : una infección generalizada a Gram negativo con derrame hemático prolongado, que se resolvió con reposición y tratamiento antibiótico, y dos infecciones de la herida posterior que sin involucrar la pared ni la cavidad torácica requirieron Toilette posterior hasta el plano del implante y tratamiento antibiótico prolongado. Ningún paciente requirió revisión de la cavidad ni pared torácica (Gráfico 2).

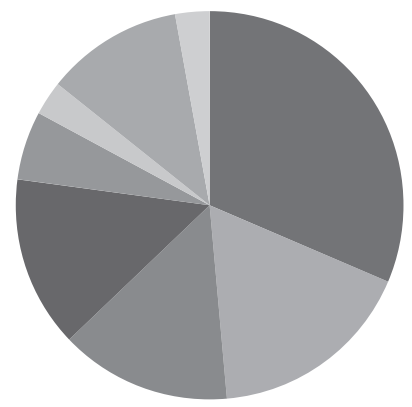

$$
\begin{aligned}
& \text { - Genética } \\
& \text { — Idiopática } \\
& \text { - Neurol } \\
& \text { - Congen } \\
& \text { - Fracturas } \\
& \text { - Hernia } \\
& \text { - Tumor } \\
& \text { - Infeccion }
\end{aligned}
$$

Gráfico 1 -Etiología

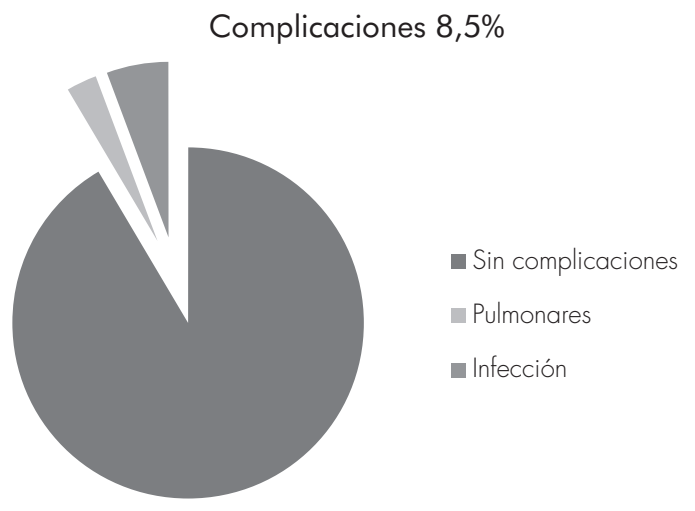

Gráfico 2 - Complicaciones

\section{DISCUSIÓN}

Tradicionalmente se utiliza la toracotomía amplia o limitada como estándar de abordaje torácico. Su realización es técnicamente sencilla y sus versiones amplias brindan una excelente visualización y acceso, pero implican en una grave agresión a la integridad de la pared torácica en todos sus planos. Por otro lado, realizar una toracotomía en los niveles torácicos altos por encima de torácica quinta, tiene a la escápula como barrera natural, que debe desplazarse. Para ese objetivo, es necesario seccionar prácticamente toda la musculatura peri-escapular medial e inferior, creando morbilidad estética y funcional apreciable.

En la búsqueda de alternativas a la toracotomía, surge la toracoscopia videoasistida de columna Blackman RG 19932. Fue realizada una etapa experimental inicial en $\operatorname{porcinos}^{8}$, posteriormente de aplicación clínica ${ }^{6}$ y finalmente se demonstró una significativa disminución en las complicaciones asociadas al abordaje, fundamentalmente, aquellas vinculadas directamente a la pared torácica ${ }^{9}$.

La anestesia asociada a la toracoscopia requiere intubación bronquial selectiva y colapso de un pulmón, incrementando la complejidad anestésica. Su utilización en pacientes con desventajas respiratorias previas, por períodos prolongados, dificulta en mantener niveles adecuados de gases en sangre durante el procedimiento y, muchas veces, es disparador de complicaciones postoperatorias vinculadas al parénquima pulmonar.

También con la toracoscopia, aun con una adecuada visualización, tenemos dificultades técnicas en alcanzar cómodamente los niveles torácicos altos porque la escápula bloquea la proyección natural de los accesos ideales requeridos, y la posibilidad de trabajar instrumentalmente en el plano de la vértebra. Para ello es necesario ingresar por el hueco supraclavicular o por la axila, incrementando el riesgo de lesiones vasculares o nerviosas.

Originalmente fue desarrollada esta vía alternativa (toracotomía posterior) para casos que requieren acceder a la columna torácica alta, donde las opciones abiertas tradicionales, o la toracoscopia, no satisfacen por su agresividad o dificultad de trabajo. La referencia bibliográfica que la inspiró y, se aproxima levemente, es la descripta por Turner y $\mathrm{Webb}^{7}$, pero esta vía, en su descripción original, no utiliza una incisión mediana y el acceso al tórax es más agresivo al seccionar músculos generando, 
de acuerdo a sus autores, una importante morbilidad en la pared torácica por lo que sólo la recomiendan en ancianos con patologías terminales.

Nuestra alternativa de abordaje utiliza una incisión mediana posterior en sintonía con la tendencia actual de realizar procedimientos combinados desde atrás ${ }^{4,10-12}$, esta vía nos permite un ingreso directo a la cavidad torácica con escasa agresión a su estructura.

En su aplicación inicial, previa a la casuística de este trabajo, limitábamos su indicación a deformidades torácicas altas con cifosis porque las consideramos la condición más adecuada a su aplicación, realizando toracotomías posteriores de un solo nivel para objetivos de dos o tres espacios. Su práctica habitual nos llevó rápidamente a extender su aplicación a toda la columna torácica en cualquier condición, patología y edad.

Por otro lado, la posibilidad de realizar múltiples toracotomías mínimas posteriores con resección costal mínima, o sin ella, la convierten en una vía fácilmente realizable, pudiendo abarcar muchos niveles y modificar el plan original a la demanda del procedimiento. Esta opción es complicada cuando la vía es una toracotomía y nos vemos en la necesidad de alcanzar un disco o cuerpo vertebral inmediatamente por encima del nivel costal abordado o muy por debajo del mismo.

Los requerimientos técnicos anestésicos e instrumentales para la toracotomía posterior son los mismos que para la toracotomía convencional; no es necesaria intubación selectiva ni instrumental sofisticado. Además, sus escasas complicaciones la colocan, en nuestra opinión, como un recurso de mucha utilidad, indispensable en las opciones del cirujano espinal (constituyendo el estándar actual de nuestro servicio).
Las limitaciones de la vía respecto a la toracotomía son un menor control visual del contralateral al lado abordado, la menor exhibición panorámica a la que brinda la toracotomía convencional, la ventana de abordaje en cada sector es más pequeña pero perfectamente focalizada. Otro aspecto cuestionable es que la incisión medial posterior debe ser al menos de T3 a T10 porque es necesario, por un lado, alcanzar la totalidad de la inserción medial del trapecio para movilizar la escápula y, además, retraer lateralmente el borde cutáneo como cuerda de arco para exponer la pared costal. Por otro lado, si no se realizan los dos tiempos el mismo día; la reintervención cercana en la misma incisión podría conjeturalmente incrementar las posibilidades de infección del tiempo posterior.

Si bien, el objetivo de este trabajo no es comparativo, hay otro trabajo nuestro de casuística similar que compara complicaciones entre toracotomía (48\%) y toracoscopia $(22 \%)^{9}$. Grossfeld et al. ${ }^{5}$ refieren complicaciones mayores en el 7,5\% y menores en el $33 \%$ de 599 vías anteriores en niños, incrementando significativamente estos valores en procedimientos combinados; ambos trabajos reflejan índices de complicación superiores a nuestra casuística con toracotomía posterior.

\section{CONCLUSIÓN}

La toracotomía posterior es un abordaje de elección en columna torácica alta y alternativa para el resto del tórax. Esta es aplicable a distintas patologías, deformidades y edades. Permite realizar procedimientos combinados mediante una única incisión cutánea posterior y su índice de complicaciones es bajo.

\section{REFERÊNCIAS}

1. Hogdson AR, Stock FE. Anterior spinal fusion for treatment of tuberculosis of the spine: the operative findings and results of treatments in the first one hundred cases. J Bone Joint Surg Br. 1960;42:295-310.

2. Blackman RG, Picetti G III, O’Neal K. Preliminary results of endoscopic procedures on the anterior thoracic spine. North American Spine Society (NASS). 10th Annual Meeting, October 18-21, Washington, DC; 1995. p. 163.

3. Capener N. The evolution of the lateral rachotomy. J Bone Joint Surg B. 1954;36:173-9.

4. Graham AW 3rd, Mac Millan M, Fessler RG. Lateral extracavitary approach to the thoracic and thoracolumbar spine. Orthopedics. 1997;20(7):605-10.

5. Grossfeld S, Winter RB, Lonstein JE, Denis F, Leonard A, Johnson L. Complications of Anterior Spinal Surgery in Children. J Pediatr Orthop. 1997;17(1):89-95.
6. Noel MA, Tello CA, Bersusky E, Francheri AL. Artrodesis anterior, toracoscopia en escoliosis. Rev Asoc Argent Ortop Traumatol. 1997;62(1):70-7.

7. Turner PL, Webb JK. A surgical approach to the upper thoracic spine. J Bone Joint Surg B. 1987;69(4):542-4.

8. Noel MA, Tello CA, Bersusky E, Francheri AL. Cirugía endoscopica espinal en porcinos. Rev Asoc Argent Ortop Traumatol. 1996;61(4):470.

9. Noël MA, Tello CA, Bersusky ES, Francheri AI, Alvarado J, Lippenholtz MC. Estudio comparativo entre la toracoscopía videoasistida y la toracotomía convencional para la artrodesis vertebral anterior en el tratamiento de escoliosis en niños y adolescentes. Bol Acad Nac Med. 2000;78(2):502.

10. Street J, Fisher C, Street J, Fisher C, Sparkes J, Boyd M, et al. Single-stage posterolateral vertebrectomy for the management of metastatic disease of the thoracic and lumbar spine. A prospective study of an evolving surgical technique. J Spinal Disord Tech. 2007;20(7):509-20.
11.Klimo P Jr, Dailey AT, Fessler RG. Posterior surgical approaches and outcomes in metastatic spinedisease. Neurosurg Clin North Am. 2004;15(4):425-35.

12. Smith JT, Gollogly S, Dunn HK. Simultaneous anteriorposterior approach through a costotransversectomy for the treatment of congenital kyphosis and acquired kyphoscoliotic deformities. J Bone Joint Surg. 2005;87(10):2281-9.

\section{Correspondencia:}

Mariano Augusto Noel

Hospital Nacional de Pediatria Dr. Profesor J. P. Garrahan

Larrea 10071 B Código Postal 1061

Ciudad Autónoma de Buenos Aires, Argentina

Tel: +05411-1545368614

E-mail:mnoelar@yahoo.com.ar 